\title{
miR-965 controls cell proliferation and migration during tissue morphogenesis in the Drosophila abdomen
}

\author{
Pushpa Verma', Stephen M Cohen ${ }^{1,2 *}$ \\ ${ }^{1}$ Institute of Molecular and Cell Biology, Singapore, Singapore; ${ }^{2}$ Department of \\ Cellular and Molecular Medicine, University of Copenhagen, Copenhagen, Denmark
}

\begin{abstract}
Formation of the Drosophila adult abdomen involves a process of tissue replacement in which larval epidermal cells are replaced by adult cells. The progenitors of the adult epidermis are specified during embryogenesis and, unlike the imaginal discs that make up the thoracic and head segments, they remain quiescent during larval development. During pupal development, the abdominal histoblast cells proliferate and migrate to replace the larval epidermis. Here, we provide evidence that the microRNA, miR-965, acts via string and wingless to control histoblast proliferation and migration. Ecdysone signaling downregulates miR-965 at the onset of pupariation, linking activation of the histoblast nests to the hormonal control of metamorphosis. Replacement of the larval epidermis by adult epidermal progenitors involves regulation of both cell-intrinsic events and cell communication. By regulating both cell proliferation and cell migration, miR-965 contributes to the robustness of this morphogenetic system.
\end{abstract}

DOI: 10.7554/eLife.07389.001

*For correspondence: scohen@ sund.ku.dk

Competing interests: The authors declare that no competing interests exist.

Funding: See page 17

Received: 09 March 2015

Accepted: 29 July 2015

Published: 30 July 2015

Reviewing editor: Matthew Freeman, University of Oxford, United Kingdom

Copyright Verma and Cohen. This article is distributed under the terms of the Creative

Commons Attribution License, which permits unrestricted use and redistribution provided that the original author and source are credited.

\section{Introduction}

Tissue morphogenesis is a complex process, through which the organism coordinates cell proliferation and cell death with cell migration and rearrangements to achieve final organ shape and size. Mechanisms controlling these processes play important role in morphogenesis, tissue repair and regeneration, and in cancer (Friedl and Gilmour, 2009; Rorth, 2009). The abdominal epithelium of Drosophila provides a useful model system in which to study the dynamics of tissue morphogenesis in vivo and to explore the genetic and cellular mechanisms that control these complex morphogenetic processes.

During metamorphosis, larval epidermal tissues undergo cellular restructuring and rearrangement to give rise to adult abdominal epithelium. The adult abdominal epithelium is produced from progenitor cells, known as histoblast cells. Histoblasts are small diploid cells, easily distinguishable from the large polyploid larval epidermal cells (LEC) that surround them. Histoblasts are specified in the embryo and lie quiescent throughout larval development (Guerra et al., 1973; Simcox et al., 1991). There are four pairs of histoblast nests in each segment, which merge to assemble the adult abdominal epidermis (Madhavan and Schneiderman, 1977). The anterior and posterior dorsal histoblast nests give rise to the external dorsal cuticle of the abdominal segments (tergites), while the ventral pair give rise to the ventral cuticle (sternites).

During larval stages, histoblasts are arrested in the G2 phase of the cell cycle. At pupariation, an ecdysone pulse triggers the expression of string (cdc25), which activates cyclin/CDK and pushes the histoblasts into rapid proliferation (Edgar and O'Farrell, 1990; Gautier et al., 1991). The proliferative phase is divided into two stages with distinct features (Madhavan and Madhavan, 1980; Ninov et al., 2009). The early 'division phase' is characterized by rapid and synchronous cell division without 
eLife digest Tissues in living organisms are shaped via complex processes that are collectively called 'morphogenesis'. Many researchers have used the fruit fly Drosophila as a model to understand morphogenesis, which occurs both during the development of a Drosophila embryo and during metamorphosis (when the pupa changes to become an adult fly).

Like other insects, adult fruit flies have three main body sections (a head, a thorax and an abdomen), which are further divided into segments. The adult's abdomen forms inside the pupa from precursor cells called histoblasts. These cells are unusual in that they develop in the embryo but remain inactive during the larval stages of life. During pupation, these cells are reactivated by a hormone called ecdysone, and gradually replace the larva's tissues. However, it was not clear how this process was coordinated.

Verma and Cohen have now demonstrated that a small RNA molecule-a microRNA called miR965-acts in histoblast cells and controls how much these cells divide as well as how they migrate during morphogenesis to replace the larval cells. MicroRNAs regulate other RNAs, called messenger RNAs, typically by targeting them for destruction. This prevents the messenger RNA molecules from being used to make proteins. When flies develop without miR-965, they are mostly normal but have defects in the formation of segments in the abdomen.

Verma and Cohen revealed that miR-965 acts by targeting two important messenger RNAs for destruction. These messenger RNAs encode a protein called String, which regulates histoblast proliferation, and another protein called Wingless. Once the pupa starts to form, the ecdysone hormone reduces the production of miR-965 to increase histoblast proliferation and migration. The miR-965 microRNA in turn reduces the level of ecdysone receptor. The ecdysone hormone acts as an all-or-nothing switch to make an irreversible change from the larval to the pupal stage. The hormone boosts its own activity by increasing expression of its own receptor. This 'positive feedback loop' acts like a switch and is very sensitive to small changes in the amount of hormone present.

Verma and Cohen propose that by reducing the levels of the hormone receptor, miR-965 makes the system more stable. This is because the hormone must first overcome the action of miR-965 before it can kick off the positive feedback loop. This takes time, and means that any change in the amount of hormone must be around for a while to have an effect. This mechanism buffers against short-lived, small changes in hormone levels that might throw the switch at the wrong time-a feature known as 'robustness'. This seems to be a complicated process to go from one state to another (i.e., from a larva to a pupa). But, the existence of the many distinct checks and balances makes sure the switch is thrown only when it is needed.

DOI: 10.7554/eLife.07389.002

intervening G2 phases to allow for cell growth. Cells double in number and decrease in size with each division, with little change in the size of the nests. This is followed by a phase of slower division, from 15-40 hr of pupal development, in which proliferation is accompanied by longer intervening gap phases to allow cell growth. During this phase, epidermal growth factor receptor (EGFR) and Insulin receptor/PI3K- signaling coordinate the growth of cells with proliferation (Ninov et al., 2009). During the growth phase, the histoblast nests begin to spread to replace the LEC. The expansion of the histoblast nests by cell migration is accompanied by programmed cell death, so that larval cells are replaced by the expanding histoblast population to maintain integrity of the epithelium (Bischoff and Cseresnyes, 2009; Nakajima et al., 2011).

Patterning of the adult abdominal segments makes use of many of the signaling pathways that pattern the embryonic and larval segments, including hedgehog, wingless, decapentaplegic and EGFR. Interactions between morphogen gradients produced by some of these proteins determine anterior-posterior and dorsal-ventral patterning of segments (Sanicola et al., 1995; Shirras and Couso, 1996; Struhl et al., 1997; Kopp et al., 1999; Ninov et al., 2010). Although abdominal segmental patterning has been extensively studied, the molecular mechanisms regulating cell division, migration, cell replacement and their interactions during formation of segments remain less well understood. Here, we provide evidence that the microRNA, miR-965, is required in the histoblast nests, where it acts via regulation of string and wingless to control histoblast proliferation and migration during pupal morphogenesis. 


\section{Results}

The miR-965 microRNA is located in the first intron of the kismet gene (Figure 1A). kismet and miR-965 are transcribed in the same direction. Quantitative PCR showed that a series of P-element insertional mutants near the first exon of the kismet gene reduced the level of mature miR-965 miRNA, suggesting that kismet and miR-965 arise from a single transcription unit (Figure 1-figure supplement 1).

To explore the biological functions of miR-965, independently from those of kismet, we produced targeted deletion mutants to remove the miRNA from the kismet intron (Figure 1A; [Chen et al., 2014]). One mutant allele was made by homologous recombination using the pw25 vector (Gong and Golic, 2003). In this allele, the genomic region containing the miRNA hairpin was replaced by a miniwhite reporter gene flanked by LoxP sites (Figure 1A, w+KO1). The presence of the intron-containing mini-white marker in the kismet intron was expected to disrupt kismet gene function. Cre-mediated excision of the LoxP-flanked mini-white cassette produced the KO1 allele, in which the miRNA was replaced by a single LoxP site (Figure 1A, w-KO1). A second independent deletion was produced using the pRMCE vector, which was designed to allow subsequent retargeting of the locus using Recombination Mediated Cassette Exchange (RMCE) (Weng et al., 2009). The miRNA hairpin was replaced by a mini-white marker flanked by inverted attP and LoxP sites. The KO2 allele was made by
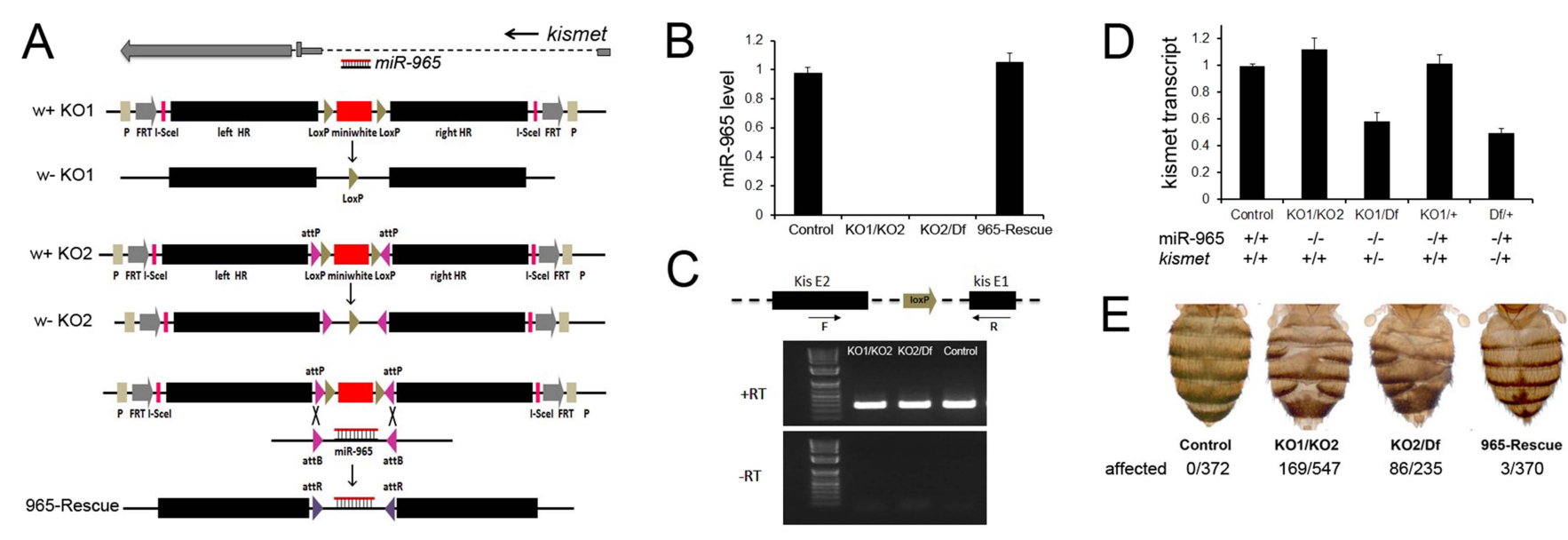

Figure 1. The miR-965 mutant. (A) miR-965 is located within the first intron of the kismet gene. The targeting strategies used to produce two independent miR-965 deletion mutants by ends-out homologous recombination are shown below. Left and Right homology arms cloned into in the targeting vector are shown in black. The w+ KO1 mutant was made by replacing miR-965 with a mini-white reporter (red) flanked by LoxP sites (grey). Sections are not represented to scale. $\mathrm{w}-\mathrm{KO} 1$ indicates the targeted allele after Cre-mediated excision of the mini-white cassette. The w+ KO2 mutant was made by replacing miR-965 with mini-white flanked by LoxP sites and inverted attP sites (pink). w-KO2 indicates the allele after Cre-mediated excision of mini-white. Use of RMCE to replace the mini-white cassette with the miRNA to produce the 965-Rescue allele is shown at bottom. (B) miR-965 RNA level measured by quantitative miRNA PCR. RNA was isolated from adult flies of the indicated genotypes. Control was $w^{1118}$. Df indicates Df(2L)ED19. Data represent the average of 3 independent experiments \pm standard deviation (SD). (C) RT-PCR using primers flanking the first intron of kismet. A PCR product of normal size was produced using RNA from flies of each of the indicated genotypes. No product was produced in the absence of reverse transcriptase. (D) Quantitative real-time RT-PCR showing kismet transcript levels in miR-965 mutants (KO1/KO2 and KO1/Df) and the heterozygous KO1/+ and $\mathrm{Df} /+$ controls. Data represent the average of 3 independent experiments \pm SD. (E) Dorsal aspect of the abdomen from females with the indicated mutant combinations. Control was $w^{1118}$. The number of affected individuals is shown below. ANOVA: $p<0.0001$ for each mutant genotype compared to the $w^{1118}$ control or to the rescued mutant.

DOI: 10.7554/eLife.07389.003

The following figure supplements are available for figure 1:

Figure supplement 1. Evidence that kismet and miR-965 arise from a common transcription unit. DOI: 10.7554/eLife.07389.004

Figure supplement 2. Phenotype classification.

DOI: 10.7554/eLife.07389.005

Figure supplement 3. Penetrance of defects in miR-965 mutants, shown as \% of affected individuals. DOI: 10.7554/eLife.07389.006 
Cre-mediated excision of the LoxP flanked mini-white reporter (Figure 1A, w-KO2). Deletion of the miRNA was verified by quantitative miRNA PCR in animals heteroallelic for the mini-white deleted versions of $\mathrm{KO} 1 / \mathrm{KO} 2$ and for the $\mathrm{KO} 2$ allele in trans to $\mathrm{Df}(2 \mathrm{~L}) \mathrm{ED} 19$, a chromosomal deletion that uncovers the kismet gene (Figure 1B). To produce a genetic rescue allele, we used RMCE to replace the mini-white cassette with a fragment containing the miR-965 hairpin (Figure 1A, 965-Rescue). miR-965 expression was restored to normal levels in the 965-Rescue allele (Figure 1B).

In the $\mathrm{w}$ - versions of the KO1 and $\mathrm{KO} 2$ alleles, DNA in the kismet intron was replaced by a single LoxP site (Figure 1A). To ask whether kismet gene function was compromised in these mini-white excised alleles, we performed molecular and genetic tests. Figure 1C shows RT-PCR to assess splicing of the first kismet intron. A PCR product of normal size was produced in RNA isolated from miR-965 ${ }^{\mathrm{w}-}$ KO1/miR-965 $5^{\mathrm{NOO} 2}$ and $\mathrm{miR}-965^{\mathrm{w}-\mathrm{KO}} / \mathrm{Df}$ animals. This qualitative assessment was confirmed by quantitative PCR (Figure 1D). kismet transcript levels were not reduced in the $\mathrm{miR}-965^{\mathrm{w}-\mathrm{KO}} / \mathrm{miR}$ $965^{\mathrm{w}-\mathrm{KO} 2}$ flies compared to controls, indicating that splicing of intron 1 was not impaired in these mutants. Genetic complementation tests also showed that the miR-965 ${ }^{\mathrm{w}-K O 1}$ and miR-965-KO2 mutant alleles were viable in trans to kismet $^{1}$, a lethal allele, as well as in trans to $D f(2 L) E D 19$, which removes kismet. Together, these data provide evidence that the mini-white excised mutant miR-965-KO1 and miR-965 ${ }^{w-K O 2}$ alleles do not compromise kismet gene function. For brevity, these mini-white deleted alleles will be referred to as $\mathrm{KO} 1$ and $\mathrm{KO} 2$ in the text and figures that follow.

\section{Abdominal segmentation defects}

Mutants lacking miR-965 had only minor effects on survival during development. Mutant adults appeared to be morphologically normal with the exception of defects in abdominal segmentation (Figure 1E). Among the affected individuals, the predominant defect was a dorsal gap in one or more abdominal segments, in some cases leading to segment fusion (Figure 1-figure supplement 2). In addition, formation of ectopic bristles associated with a polarity defect was observed in $\sim 15 \%$ of affected individuals (Figure 1-figure supplement 3). Polarity reversal was always accompanied by a gap or segment fusion phenotype. These defects were rescued by restoring miRNA expression using the miR-965 RMCE rescue allele (Figure 1E).

\section{miR-965 activity in histoblasts}

The abdominal segmentation defects observed in miR-965 mutants suggested that the miRNA might be required in the histoblasts. To visualize miR-965 activity, we made use of a sensor transgene consisting of a ubiquitously expressed GFP reporter with a perfect target site for miR-965 in the 3' UTR. Sensors of this design allow miRNA activity to be visualized by downregulation of GFP (Brennecke et al., 2003). The control sensor, lacking the miRNA-target site, was expressed at comparable levels in the large polyploid LEC and in the smaller histoblast cells (hb, Figure 2). miR-965 sensor GFP levels were lower in the histoblast nests, compared to the adjacent larval cells, particularly in the histoblast cells near the edge of the nests (Figure 2). This difference was lost when the sensor was introduced into the miR-965 mutant background, providing evidence that the reduced GFP level in the histoblasts is due to miR-965-mediated repression (Figure 2).

\section{Defects in histoblast development}

The escargot (esg) gene is expressed in histoblasts from the time of their specification in the embryo until the late pupal stage, and provides a marker to visualize histoblast development (Hayashi et al., 1993). Figure 3A presents still images taken from time-lapse videos of esg-GAL4, UAS-GFP pupae to visualize the first 3 mitotic divisions of the histoblasts. In the controls, cells became smaller and the number of cells doubled after each division (Video 1). This pattern of synchronous division was perturbed in the miR-965 mutant. Asynchronous division of miR-965 mutant histoblasts resulted in the presence of cells of different sizes (Figure 3A, Video 2). In the mutant, a subset of histoblast nuclei became pyknotic, fragmented and disappeared, indicating cell death (Video 3). Cell death was rare during this phase in the control samples. Synchronous division was restored and cell death was suppressed in the miR-965-rescue allele (Figure 3A, Video 4).

Subsequently, the rate of histoblast nest expansion was slower in the miR-965 mutant, compared to the controls (Figure 3B). Expansion of the histoblast nests was quantified in segments 3 and 4 by monitoring the speed of migration (Figure 3-figure supplement 1, Videos 5, 6). The average speed 


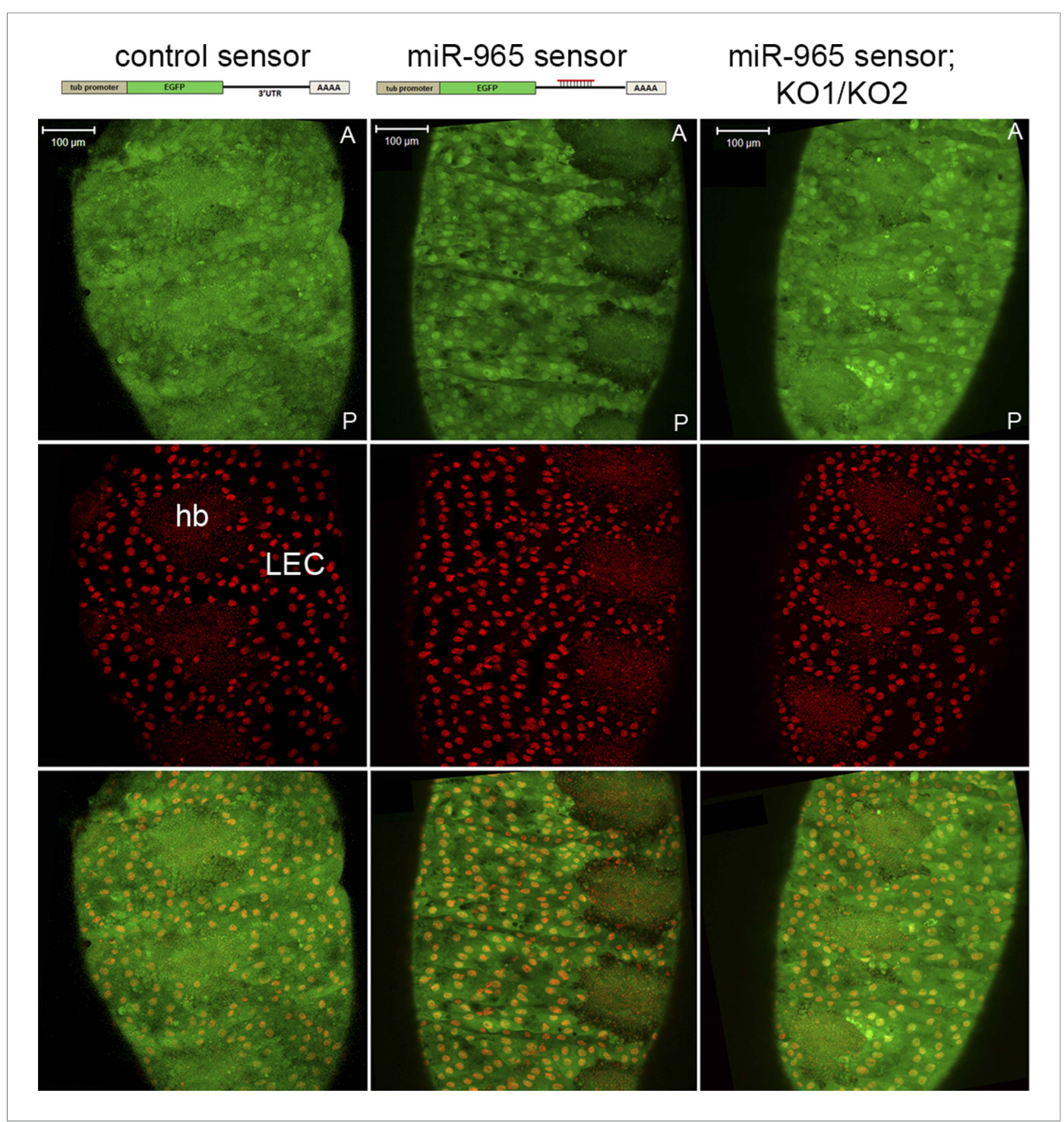

Figure 2. miR-965 expression in histoblasts. Top: design of the control and miR-965 sensor transgenes. EGFP was under control of the tubulin promoter. For the miR-965 sensor, 1 copy of a perfect miR-965 target sequence was placed into the SV40 UTR. Images showing GFP expression from the control sensor (left) and miR-965 sensor (middle) transgenes at $21 \mathrm{hr}$ APF. Histoblast nests consist of small diploid histoblast cells (hb) surrounded by large polyploid larval epidermal cells (LEC). Nuclei were labeled with histone-RFP (red). Downregulation of GFP was lost when the transgene was placed in the KO1/KO2 miR-965 mutant background (right). Anterior (A), posterior (P). Scale bar: $100 \mu \mathrm{m}$.

DOI: 10.7554/eLife.07389.007

of migration of third and fourth histoblast nests in the control samples was $15 \mu \mathrm{m} / \mathrm{hr}$, compare with $\sim 6.5 \mu \mathrm{m} / \mathrm{hr}$ in the mutant. The rate of nest expansion was increased to $12-14 \mu \mathrm{m} / \mathrm{hr}$ by restoring miR-965 expression with the rescue allele the miR-965 mutant background (Video 7 ).

LEC generally undergo programmed cell death as the histoblasts nests start to grow (Nakajima et al., 2011). In miR-965 mutants, we observed that some of these cells were still present in the nests, suggesting a failure to eliminate LECs in the mutant (Figure 3-figure supplement 2). Delayed expansion of the histoblast nests, combined with the persistence of LECs, is likely to be responsible for the gaps observed in adult abdominal segments.

It was noted that the migration defects observed in the videos seem to be more severe than the segmentation defects observed in the adult flies. However, we did not observe significant preeclosion lethality (Figure 3-figure supplement 3). Given that there was no evidence for loss of 

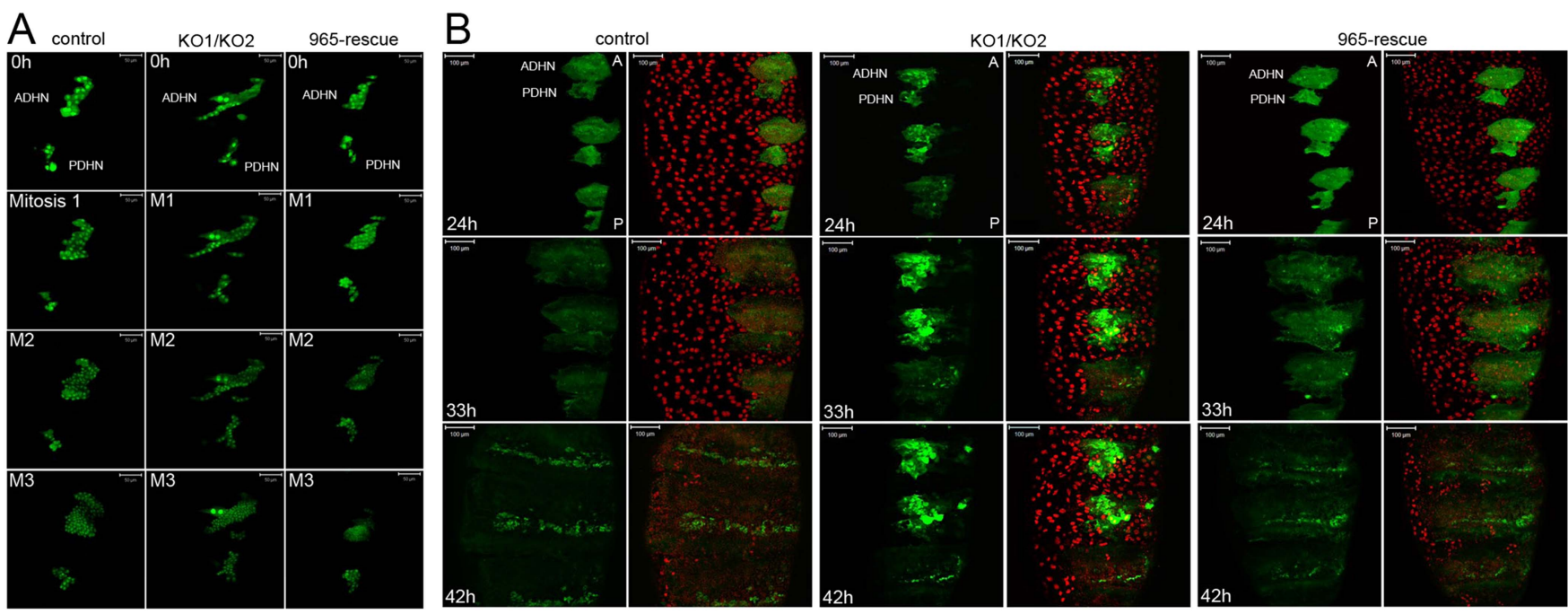

Figure 3. Abnormal histoblast proliferation and migration in the miR-965 mutant. (A) Still images taken from time-lapse videos of control, miR-965 mutant (KO1/KO2) and rescued mutant showing the reduction divisions of the early histoblast proliferation phase. M1, M2 and M3 indicate images taken after mitosis 1, 2 or 3. Imaging was started 0-1 hr APF. Histoblasts were labeled by esg-Gal4 directed expression of UAS-nuclear GFP. ADHN and PDHN represent anterior dorsal histoblast nests and posterior dorsal histoblast nests. Scale bars: $50 \mu \mathrm{m}$. Note the different cell sizes in the miR-965 mutant histoblast nests. (B) Still images taken from time-lapse videos at 24, 33 and $42 \mathrm{hr}$ APF from control, miR-965 mutant and rescued mutant to illustrate expansion of the histoblast nests to replace LECs. Histoblasts were labeled by esg-Gal4 directed expression of cytoplasmic GFP. esg-GAL4 and UAS-GFP were recombined onto the miR-965 mutant and onto the miR-965 Rescue chromosome. Nuclei were labeled red with H2-RFP A and P indicate anterior and posterior orientation. Scale bars: $100 \mu \mathrm{m}$.

DOI: 10.7554/eLife.07389.008

The following figure supplements are available for figure 3 :

Figure supplement 1. Rate of histoblast nest expansion measured from time-lapse videos.

DOI: 10.7554/eLife.07389.009

Figure supplement 2. Large polyploid cells in miR-965 mutant histoblast nests

DOI: 10.7554/eLife.07389.010

Figure supplement 3. Pupal survival assays.

DOI: 10.7554/eLife.07389.011

a class of more severely affected animals, we suggest that the apparent difference reflects a delay in tissue replacement in the mutant, so that most animals end up with milder defects by the end of pupariation than were apparent during the early pupal time-window in the videos.

\section{miR-965 regulates string and wingless}

miR-965 is predicted to target 69 genes (www.targetscan.org). polycomb, string, wingless, homothorax, Tor, Hsp83 and jumeau were selected for further analysis based on the quality of the predicted target site and on Flybase annotation suggesting roles in segmentation. Of these, only string and wingless $(\mathrm{wg}) \mathrm{mRNAs}$ were upregulated in RNA isolated from miR-965 mutant pupae, and were also restored to near normal levels in the rescued mutant (Figure 4A,B), as would be expected for functional miRNA targets.

The wg $3^{\prime}$ UTR was used to make a luciferase reporter transgene and tested for regulation by miR-965 in S2 cells. miR-965 expression reduced wg 3'UTR reporter activity, and this regulation was lost in the wg UTR reporter mutated to disrupt pairing with the miRNA seed sequence (Figure $4 C$, mutated residues shown in red). miR-965 expression also reduced expression of the string $3^{\prime}$ UTR luciferase reporter (Figure 4D). Mutation of the predicted target site to disrupt seed pairing partially offset regulation of the string $3^{\prime}$ UTR luciferase reporter (Figure 4D, mutated residues in red). We noted the presence of a second potential target site nearby (Figure 4-figure supplement 1). More extensive mutation to disrupt both sites further compromised regulation by miR-965 (Figure 4D). 


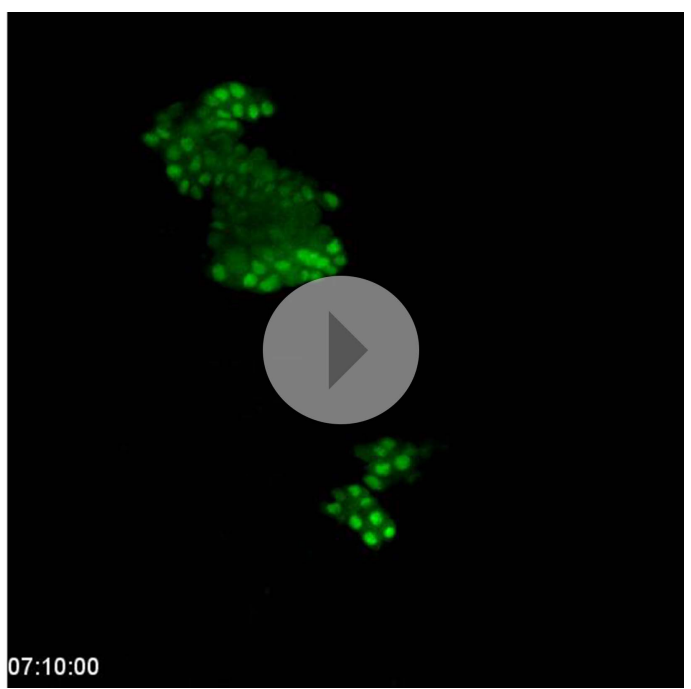

Video 1. Control, division phase. Early synchronous divisions of histoblast nests in Esg-GAL4>UAS-nuclear GFP controls. GFP was used to track anterior and posterior dorsal histoblast nests during the early division phase. Animals were collected for imaging at $0 \mathrm{hr}$ APF, the white pre-pupal stage. ADHN: Anterior dorsal histoblast nest. PDHN: posterior dorsal histoblast nest. $A$ and $P$ indicate anterior and posterior orientation. Scale bar: $50 \mu \mathrm{M}$. Refers to Figure $\mathbf{3 A}$. DOI: 10.7554/eLife.07389.012

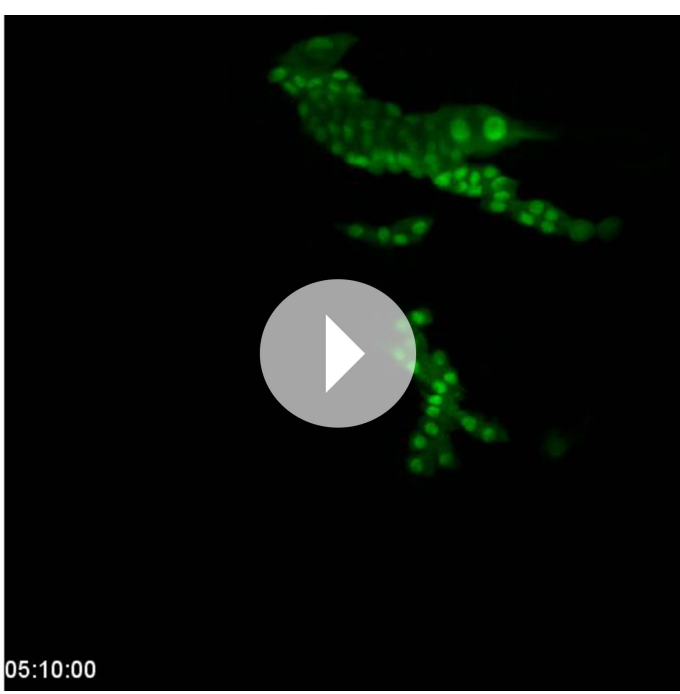

Video 2. miR-965 mutant division phase. Early asynchronous divisions in the miR-965 mutant (KO2, esgGAL4>UAS-nuclear GFP/KO1). Esg-GAL4>UAS-nuclear GFP was recombined onto miR-965 RMCE mutant (KO2) chromosome. ADHN and PDHN indicate anterior and posterior dorsal histoblast nests. Animals were collected for imaging at $0 \mathrm{hr}$ APF. Scale bar: $50 \mu \mathrm{M}$. Refers to Figure 3A.

DOI: 10.7554/eLife.07389.013

Other non-canonical target sites might be responsible for the remaining regulation by miR-965, however, we do not exclude the possibility that there could also be indirect effects of miR-965 on expression of this reporter. These findings provide evidence that miR-965 can regulate expression of string and wg.

\section{Contributions of string and wingless to the miR-965 mutant phenotype}

To ask whether the increases in string and wg expression might be responsible for the miR-965 mutant phenotype, we first asked if overexpressing them in an otherwise normal genetic background could phenocopy the mutant. esg-Gal4 driven expression of a UAS-string transgene produced abdominal segment gaps, segment fusion and polarity reversal phenotypes, similar to those observed in the miR965 mutant (Figure 5A Figure 5-figure supplement 1). The proportion of flies with defects caused by string overexpression was similar to that in the miR-965 mutant (Figure 5B). string overexpression caused asynchronous histoblast division and apoptosis during the early division phase (Figure 5-figure supplement 2, Video 8). Expansion of the string-overexpressing histoblast nests was slowed, and the histoblasts were unable to fully replace the LECs (Figure 5-figure supplements 2, 3, Video 9), similar to what was observed in miR-965 mutants. Thus, string overexpression was sufficient to reproduce the miR-965 mutant phenotype.

To assess the contribution of wg overexpression, we made use of a UAS transgene directing expression of a temperature sensitive form of Wg protein (Wilder and Perrimon, 1995). Use of Wg ${ }^{\text {ts }}$ was required to allow stage specific activation of $\mathrm{Wg}$ in the histoblasts. Continuous expression of wild-type Wg under esg-Gal4 control was lethal. $\mathrm{Wg}^{\text {ts }}$ protein is inactive at $25^{\circ} \mathrm{C}$. Flies carrying esg-Gal4 and UAS$\mathrm{wg}^{\text {ts }}$ were reared at $25^{\circ} \mathrm{C}$ or shifted to $18^{\circ} \mathrm{C}$ in the third larval instar to allow expression of active $\mathrm{Wg}$ as the histoblasts began proliferation. This resulted in a phenocopy of the segment gap phenotype in $7 \%$ of animals (Figure $5 \mathrm{C}$ ). Phenocopy was rare in the animals raised continuously at $25^{\circ} \mathrm{C}$ to maintain low $\mathrm{Wg}$ activity $\left(0.4 \%\right.$ affected, Figure $5 C ; p=0.014$ comparing UAS-wg ${ }^{\text {ts }}$ at $18^{\circ}$ vs $25^{\circ}$, Fisher's exact test).

Next we asked whether limiting target gene overexpression could suppress the mutant phenotype. Lowering string activity by introducing string mutant alleles reduced the penetrance of the segment gap 


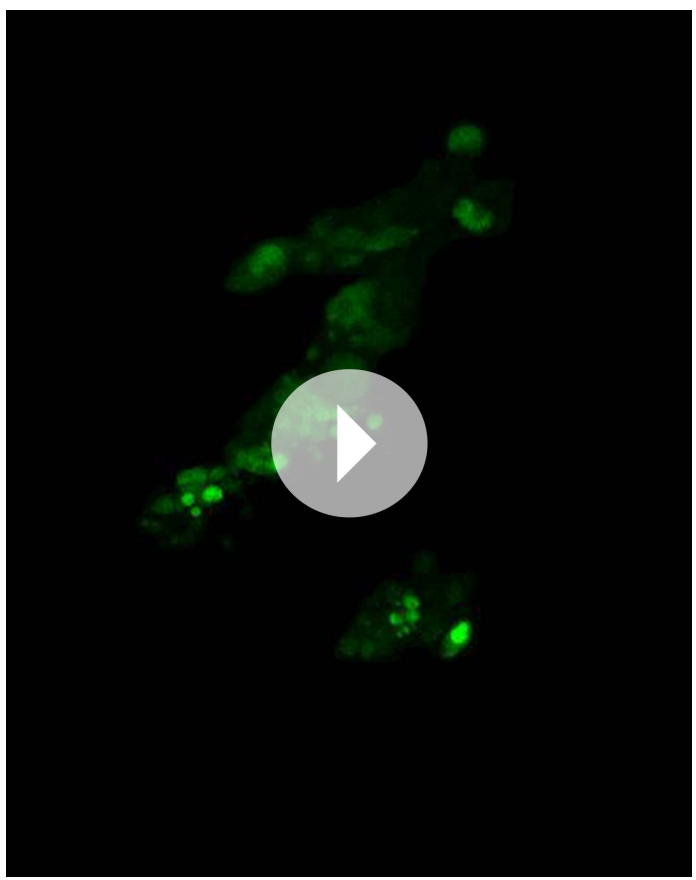

Video 3. miR-965 mutant apoptosis. Apoptotic cells are seen during the early histoblast division phase in the miR-965 mutant. Esg-GAL4>UAS-nuclear GFP was recombined onto miR-965 RMCE mutant (KO2) chromosome. ADHN and PDHN indicate anterior and posterior dorsal histoblast nests. Animals were collected for imaging at $0 \mathrm{hr}$ APF. Scale bar: $50 \mu \mathrm{M}$. Refers to Figure 3A.

DOI: 10.7554/eLife.07389.014

cell migration phenotype of the miR-965 mutant (Figure 5-figure supplements 4, 5, Video 11)

Wingless protein $(\mathrm{Wg})$ is expressed in the anterior dorsal histoblast nests, during their growth and migration (Kopp et al., 1999). The level of Wg protein was higher in the miR-965 mutant (Figure 5E). $\mathrm{Wg}$ is a secreted protein, and its distribution appears to be broader, reaching some of the LECs in the mutant, perhaps reflecting the higher level of protein produced (Figure 5E). To ask whether this elevated Wg expression contributes to the defects in the mutant, we introduced wg mutant alleles into the miR-965 (KO1/KO2) mutant background. The segment gap phenotype was reduced comparing

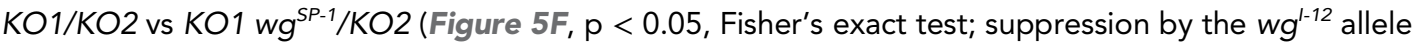
was not statistically significant). Next, we tested the effect of removing one copy each of stg and wg. The segment gap phenotype was reduced comparing $\mathrm{KO} 1 / \mathrm{KO} 2 \mathrm{vs} \mathrm{KO} 1 \mathrm{wg}^{\mathrm{SP}-1} / \mathrm{KO} 2 ; \mathrm{stg}^{4} /+$ (Figure $5 \mathrm{~F}$, $\mathrm{p}<0.001$, Fisher's exact test). Taking out one copy each of stg and wg lowered penetrance of the phenotype to $4 \%$, compared with $6-7 \%$ for stg/+ alone or $12 \%$ for $w g /+$ alone.

These experiments provide evidence that overexpression of string and wg each contribute to the miR-965 mutant histoblast phenotypes. The effect of limiting Wg expression in the mutant background may appear to be smaller than that of limiting String. However, to make a meaningful comparison of the relative contribution of these two targets, it would be necessary to restore each of them to normal levels. The genetic method used to reduce target activity in the mutant background does not allow precise control over the final target level, so this question cannot be addressed. We also do not exclude the possibility that there could be other functionally significant targets in addition to $\mathrm{Wg}$ and String.

\section{Ecdysone mediated regulation of miR-965}

Ecdysone pulses at the beginning of pupariation have been shown to induce string expression in order to reactive histoblast proliferation (Ninov et al., 2009). In light of the relationship between miR-965 


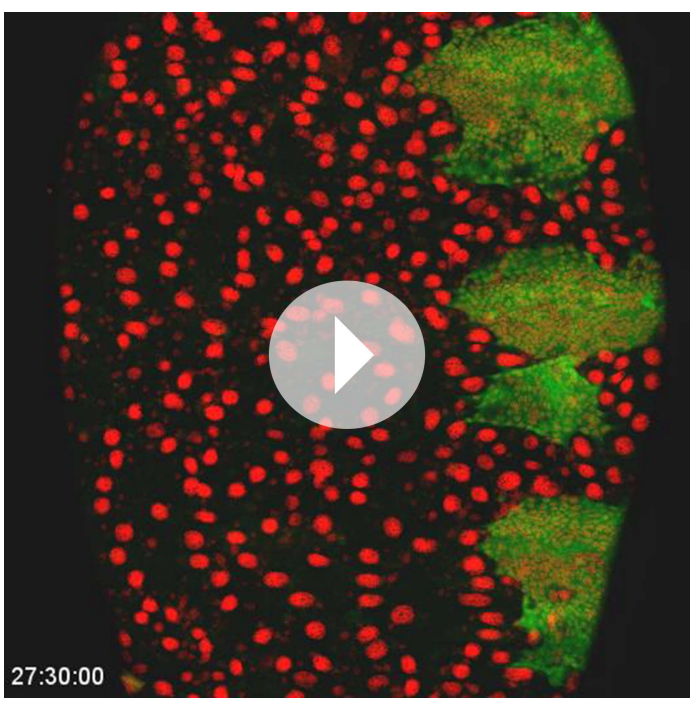

Video 5. Control, growth phase. Migration of histoblast nests during the growth phase in an EsgGAL4>UAS-cytoplasmic GFP control pupa. GFP (green) is used to monitor growth and migration of histoblast nests. H2-RFP (red) marks the nuclei. Big nuclei are LECs and small nuclei are histoblast cells. ADHN and PDHN indicate anterior and posterior dorsal histoblast nests. Scale bar: $100 \mu \mathrm{M}$. Refers to Figure 3-figure supplement 1.

DOI: 10.7554/eLife.07389.016

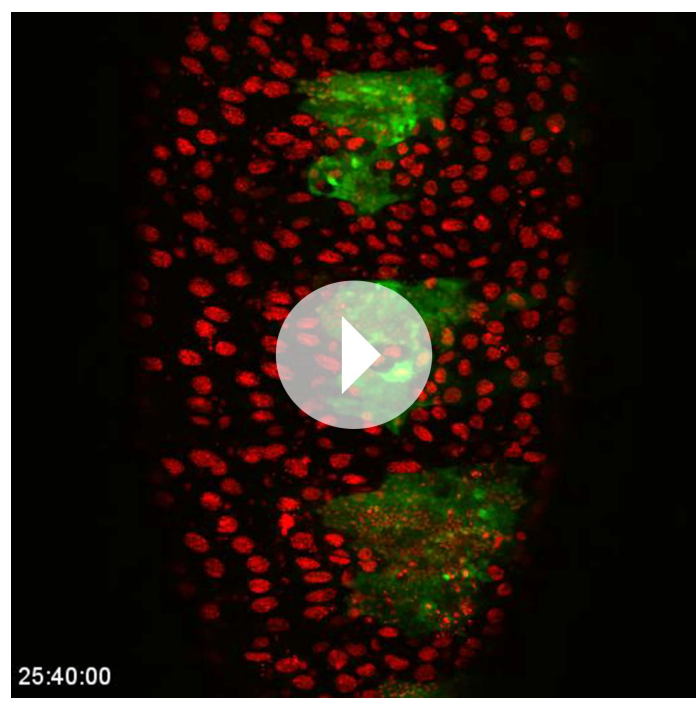

Video 6. miR-965 mutant growth phase. Delayed migration of histoblast nests during the growth phase in the miR-965 mutant. Esg-GAL4>UAS-cytoplasmic GFP was recombined onto both $\mathrm{KO} 1$ and $\mathrm{KO} 2$ mutant chromosomes. This video shows a KO2, esg-

GAL4>UAS-cytoplasmic GFP/KO1 pupa. H2-RFP (red) marks the nuclei. ADHN and PDHN indicate anterior and posterior dorsal histoblast nests. Scale bar: $100 \mu \mathrm{M}$. Refers to Figure 3-figure supplement 1.

DOI: 10.7554/eLife.07389.017

and string, we asked whether miR-965 expression might be under Ecdysone control at this stage. We made use of a UAS-EcR ${ }^{\mathrm{RNAi}}$ transgene expressed under esg-Gal4 to reduce Ecdysone receptor levels in the histoblasts. RNAi-mediated depletion of EcR mRNA led to an increase in the level of the miR965 primary transcript and mature miRNA (Figure 6A, Figure 6-figure supplement 1), and to reduced string mRNA levels in RNA samples isolated from early pupae (Figure 6A). EcR binding sites have been identified near the host gene, kismet (Gauhar et al., 2009), consistent with the possibility that ecdysone regulates expression of both kismet and miR-965. When miR-965 was overexpressed in the histoblast cells using esg-GAL4, string transcript levels were reduced (Figure 6B) and histoblast cell divisions were arrested (Figure 6C, Video 12). This phenotype resembles EcR-B mutants, in which histoblast division is compromised (Bender et al., 1997). Pupae overexpressing miR-965 in histoblast cells did not survive beyond $\sim 12$ APF, so it was not possible to monitor the later stages of histoblast migration in this genotype.

In light of the finding that EcR limits miR-965 expression, we asked whether Ecdysone signaling might act via miR-965 to regulate string. We introduced a UAS-EcR ${ }^{R N A i}$ into the miR-965 mutant background and measured the levels of string mRNAs. Depletion of EcR did not reduce string mRNA in animals lacking miR-965 (Figure 6D). This provides evidence that Ecdysone signaling is mediated through regulation of the miR-965 miRNA to regulate histoblast proliferation.

In the course of this analysis, we observed that EcR transcript levels increased in the miR-965 mutant (Figure 6D). Both mature and primary transcript levels increased, suggesting an indirect effect of the miRNA on EcR transcription. To ask if there might also be a post-transcriptional component to the regulation of EcR by miR-965, we used an EcR 3' UTR reporter transgene linked to GFP (Varghese and Cohen, 2007). GFP expression in the histoblast nests did not increase in the miRNA mutant background, indicating indirect regulation of EcR by miR-965 (Figure 6-figure supplement 2). These experiments provide evidence for a regulatory feedback relationship between miR-965 and the Ecdysone receptor (Figure 6E). EcR activity limits miR-965 expression. miR-965 activity limits EcR primary transcript levels, suggesting an effect on transcription. 


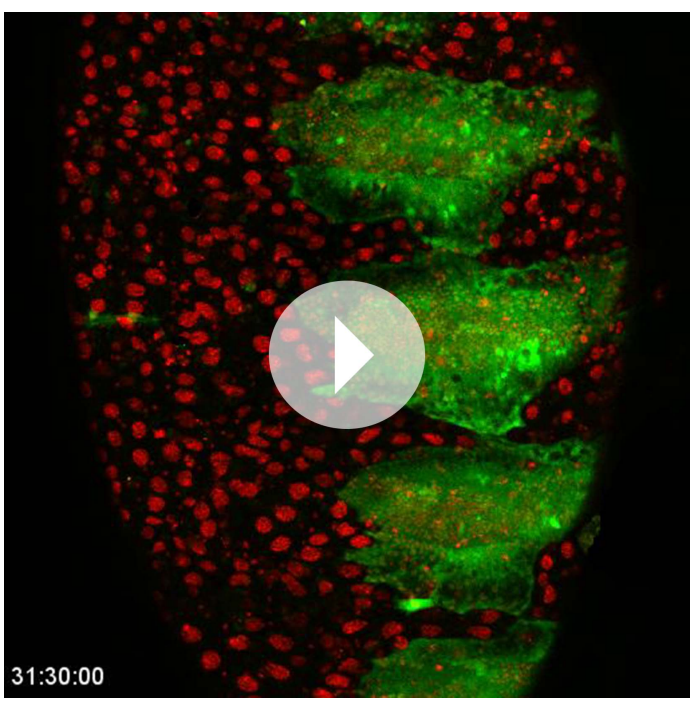

Video 7. miR-965 Rescue growth phase. Migration of histoblast nests during the growth phase in the miR-965 RMCE rescue genotype. Esg-GAL4>UAS-cytoplasmic GFP was recombined onto the miR-965 RMCE rescue chromosome. H2-RFP (red) marks the nuclei. ADHN and PDHN indicate anterior and posterior dorsal histoblast nests. Scale bar: $100 \mu \mathrm{M}$. Refers to Figure 3-figure supplement 1.

DOI: 10.7554/eLife.07389.018

stochastic fluctuation in EcR activity. Coupling EcR positive auto-feedback to miRNA-mediated repression allows a robust switch function upon Ecdysone stimulation, while protecting the system from the effects of biological noise. This study provides evidence that miR-965 plays an analogous role in regulating EcR response and suggests that miR-965 confers robustness to the EcR response in the histoblasts.

Upregulation of string in the miR-965 mutant contributes to the defects in histoblast proliferation. How misregulation of string might contribute to the migration defects is less immediately obvious. Previous work has shown that cell cycle progression in the histoblast population is required to trigger programmed cell death in the surrounding LEC (Nakajima et al., 2011). Those authors provided evidence that cell growth and the expansion of the histoblast nests may be required to elicit LEC apoptosis. Although the mechanism by which expansion of the histoblasts triggers LEC death is not clear, elevated string expression in the miR-965 mutant is likely to be responsible for the cell cycle progression defects during this phase, hindering normal LEC removal and histoblast migration.

Persistence of the LECs might also be a consequence of the increased expression of Wg protein in the mutant histoblast nests. Wg acts in combination with EGFR and Dpp signals to control abdominal segment patterning (Shirras and Couso, 1996; Kopp et al., 1999; Ninov et al., 2009, 2010). These signals are thought to control differential cell adhesion, which may be important for elimination of the LECs as well as for proper segmental fusion of the histoblast nests. Elevated expression of Wg protein may lead to an expanded range of action, perhaps resulting in ectopic Wg activity in the LECs.

Each adult abdominal segment has a well-defined anterior-posterior polarity. Wg is required from 15-20 hr APF for bristle formation and from 18-28 hr APF for tergite differentiation and pigmentation. Overexpression of $\mathrm{wg}$ has been shown to cause ectopic bristle formation, and shaggy mutant clones, which constitutively activate wg signaling, can cause polarity reversal in abdominal bristles, while EGFR, FGF, dpp and Notch signaling have no effect on the polarity of bristles in adult epidermis (Lawrence et al., 2002). Wg levels are normally higher in the posterior region of the anterior histoblast nests and lower more anteriorly. Our finding that $\mathrm{Wg}$ levels were elevated and that the distribution of $\mathrm{Wg}$ was broader than normal suggests ectopic $\mathrm{Wg}$ activity throughout the histoblast 
A

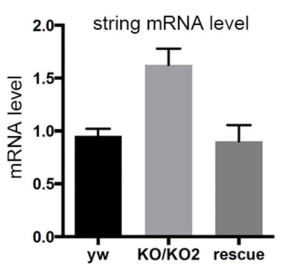

B

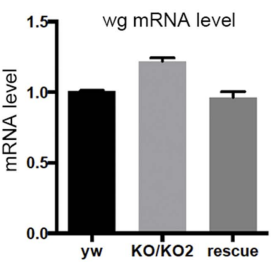

C

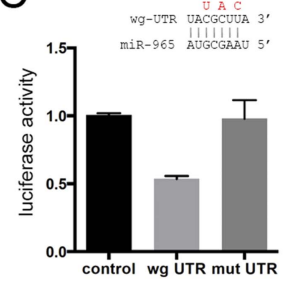

D

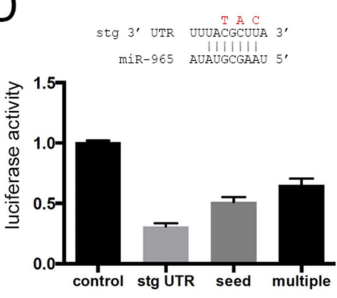

Figure 4. miR-965 regulates string and wingless. (A, B) string (stg) and wingless (wg) transcript levels measured by quantitative real time RT-PCR in RNA isolated from $w^{1118}$ control, KO1/KO2 and 965-rescue pupae at 21 hr APF. Data represent the average of three independent RNA collections \pm SD. ANOVA: $p<0.01$ comparing KO1/KO2 with control or with rescue for stg and wg. (C) Top: diagram of the predicted miR-965 target site in the wg 3' UTR, showing pairing to the miRNA seed sequence. Residues shown in red were mutated in the mutant version of the wg 3' UTR luciferase reporter. Below: luciferase activity in S2 cells transfected to express a tubulin-promoter miR-965 transgene, Renilla luciferase and the indicated firefly luciferase reporters. Control indicates the luciferase reporter with the SV40 3' UTR, which lacks miRNA binding sites. wg UTR indicates the intact full-length wg 3' UTR. Mut indicates the wg 3' UTR with the miRNA seed site mutated as indicated in red. Data represent the average of 3 independent experiments \pm SD. ANOVA: $p<0.001$ comparing control to the intact $3^{\prime}$ UTR. $p=0.001$ comparing the intact and site mutant versions of the 3' UTR. (D) Top: diagram of the predicted miR-965 target site in the stg 3' UTR, showing pairing to the miRNA seed. Residues shown in red were mutated in the seed mutant version of the reporter. The changes made in the extended target site mutant reporter are shown in Figure 3. Below: luciferase activity as in panel C. Data represent the average of 3 independent experiments \pm SD. ANOVA: $p<0.0001$ comparing control to the intact 3' UTR and comparing intact to seed mutant and multiple mutant UTR reporters.

DOI: 10.7554/eLife.07389.019

The following figure supplement is available for figure 4:

Figure supplement 1. (A) Predicted miR-965 sites in the string 3'UTR.

DOI: 10.7554/eLife.07389.020

nest, including cells that normally experience low Wg levels. Ectopic spread of Wg could be responsible for the formation of ectopic bristles and for the occasional instances of polarity reversal observed in the anterior part of tergites in the miR-965 mutants.

Replacement of the larval epidermis during metamorphosis involves regulation of both cell-intrinsic events in the abdominal histoblasts and communication between histoblasts and the larval cells they will replace. miR-965 acts on at least two separate processes required during histoblast morphogenesis. A miRNA with multiple targets can add a layer of regulation, acting across different pathways to integrate their activities (Herranz and Cohen, 2010). In doing so, the miR-965 miRNA appears to contribute to the robustness of this complex morphogenetic system.

\section{Materials and methods}

\section{Fly strains}

$W^{1118}$ was used as the control genotype unless otherwise indicated. esg-GAL4, UAS-GFP was obtained from Shigeo Hayashi. UAS-stg.N4, stg ${ }^{4}$, stg ${ }^{E Y 12388}, w^{S p-1}, w^{l-12}, K_{i s}{ }^{1}, K_{i s}{ }^{k 13416}, K_{i s^{k 11324}}, K_{i s}{ }^{k 10237}, K_{i s}^{B G 01657}$, $K_{i s}{ }^{K G 08532}, K_{i s}{ }^{E Y 12846}, D f(2 L) E x e 7702, D f(2 L) E D 19$ are from Bloomington stock center. EcR-RNAi (v37059) was from Vienna Drosophila Research Center and P\{GawB\}esg ${ }^{\text {NP7011 }}$ and ZCL2207 (Atp $\alpha$-GFP) was from DGRC, Kyoto.

\section{Mutant generation}

miR-965 (KO1) and miR-965 RMCE (KO2) deletion mutants were generated by targeted homologous recombination as described (Chen et al., 2011, 2014). Left and right homology arms were amplified by PCR from genomic DNA. The 4055 bp left homology arm was amplified with primers: 


\section{A

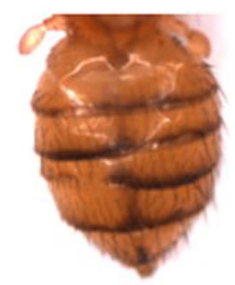

gap
esg-GAL4 + UAS-string

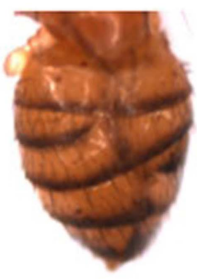

fusion

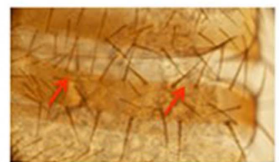

polarity reversal
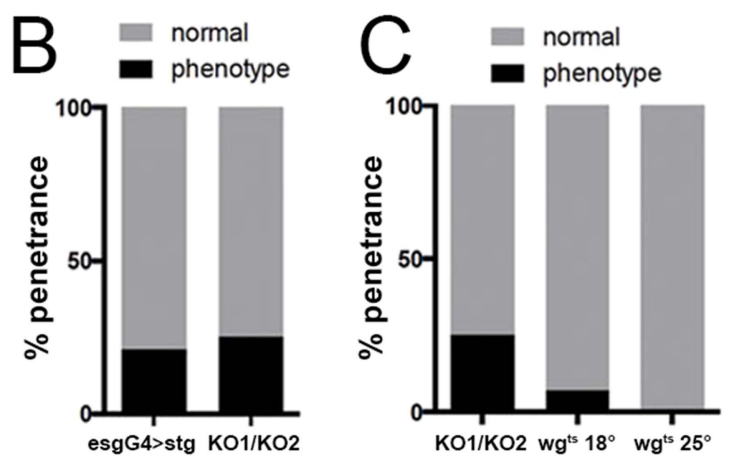

D
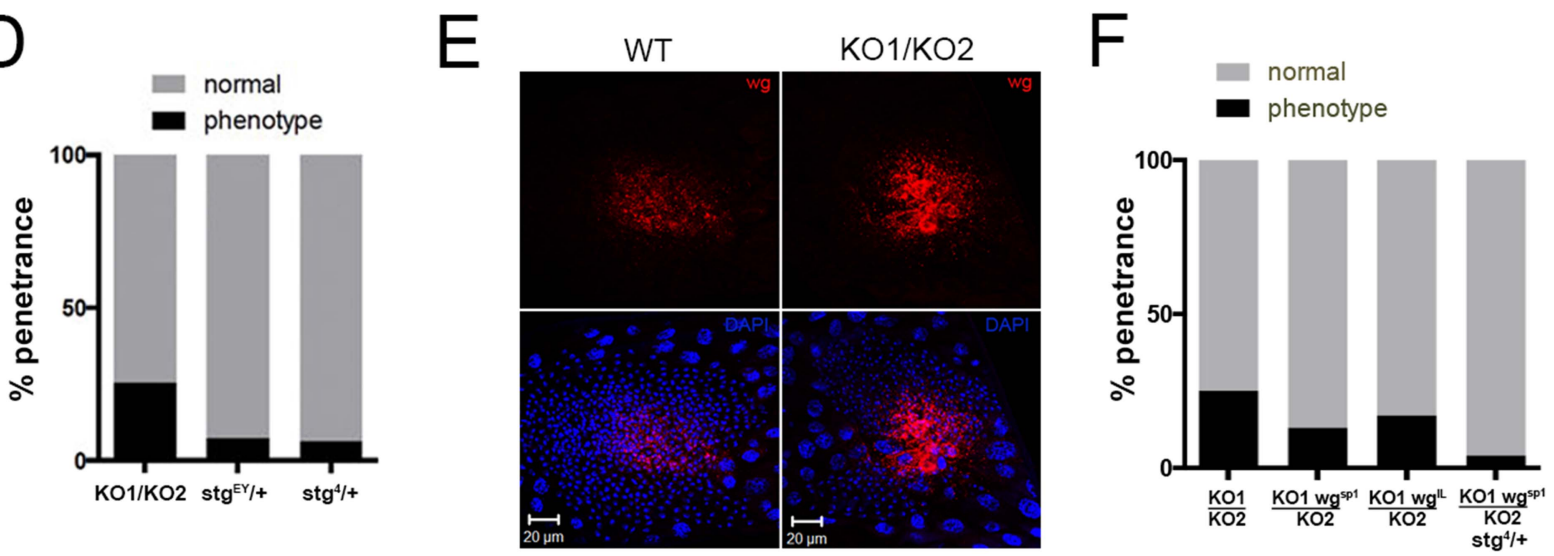

Figure 5. Overexpression of string and wg contributes to the miR-965 mutant phenotype. (A) Dorsal views of abdomens from adult female esg-Gal4 UASstring flies illustrating the segment gap, segment fusion and polarity reversal phenotypes. (B) Penetrance of abdominal defects of all classes in esg-Gal4 UAS-string vs mutant. esg-Gal4 UAS-string: $n=97 / 469 ; \mathrm{KO} 1 / \mathrm{KO} 2 \mathrm{n}=110 / 446 . p=0.16$ Fishers exact test. (C) Penetrance of abdominal defects in esgGal4 UAS-wg ${ }^{\text {ts }}$ flies reared at $18^{\circ}$ and $25^{\circ} \mathrm{C}$ vs KO1/KO2. esg-Gal4 UAS-wg ts reared at $18^{\circ} \mathrm{C}: \mathrm{n}=9 / 129$; esg-Gal4 UAS-wg ts at $25^{\circ} \mathrm{C} n=1 / 254$; $\mathrm{KO} 1 / \mathrm{KO}^{\text {ts }}$ $n=110 / 446 . p=0.014$ comparing $w^{\text {ts }}$ at 18 vs $25^{\circ} \mathrm{C}$, Fishers exact test. (D) Penetrance of abdominal defects comparing $\mathrm{KO} 1 / \mathrm{KO} 2$ mutants with $\mathrm{KO} 1 / \mathrm{KO} 2$ mutants carrying one copy of string ${ }^{E Y 12388}$ or string ${ }^{4}$ alleles. $p<0.001$ comparing KO1/KO2 to KO1/KO2; stg ${ }^{E Y} /+$ or stg $^{4} /+$ using Fisher's exact test. (E) Confocal micrographs showing dorsal histoblast nests of wild-type (WT) and miR-965 mutant (KO) at 24 hr APF labeled with anti-Wg (red). Nuclei were labeled with DAPI (blue). Scale bar: $20 \mu \mathrm{m}$. Anterior and dorsal histoblast nests in the miR-965 mutants were not yet fused at 24 hr APF, due to delayed migration. Images were captured using identical microscope settings. (F) Penetrance of abdominal segmentation defects comparing KO1/KO2 mutants with $\mathrm{KO} 1 / \mathrm{KO} 2$ mutants carrying one copy of $\mathrm{wg}^{\mathrm{SP}-1}$ or $\mathrm{wg}^{\mathrm{l}-12}$ temperature sensitive alleles or carrying one copy of $\mathrm{wg}^{\mathrm{SP}-1} \mathrm{and}^{\mathrm{stg}} \mathrm{g}^{4}$ together. $\mathrm{p}<0.05$ comparing $\mathrm{KO} 1 / \mathrm{KO} 2$ to $\mathrm{KO} 1$, wg $\mathrm{wP}^{\mathrm{SP}} / \mathrm{KO} 2$ using Fisher's exact test. $\mathrm{KO} 1 / \mathrm{KO} 2$ was not significantly different from $\mathrm{KO} 1, \mathrm{wg}^{\mathrm{l}-12} / \mathrm{KO}^{2}$, perhaps because wg $^{l-12}$ is a weaker, temperature sensitive allele. $\mathrm{p}<0.001$ comparing KO1/KO2 with KO1, wg ${ }^{\text {SP-1 }} / \mathrm{KO}^{2}$; stg $^{4} /+$ using Fisher's exact test. DOI: 10.7554/eLife.07389.021

The following figure supplements are available for figure 5:

Figure supplement 1. The proportion of flies with defects caused by string overexpression.

DOI: 10.7554/eLife.07389.022

Figure supplement 2. Still images from a time-lapse video of esg-Gal4>UAS-string histoblasts.

DOI: 10.7554/eLife.07389.023

Figure supplement 3. Speed of histoblast nest migration.

DOI: 10.7554/eLife.07389.024

Figure supplement 4. Rescue of the migration defect of miR-965 mutants with reduced levels of string. DOI: 10.7554/eLife.07389.025

Figure supplement 5. Speed of histoblast migration restored by reduced string activity.

DOI: 10.7554/eLife.07389.026 


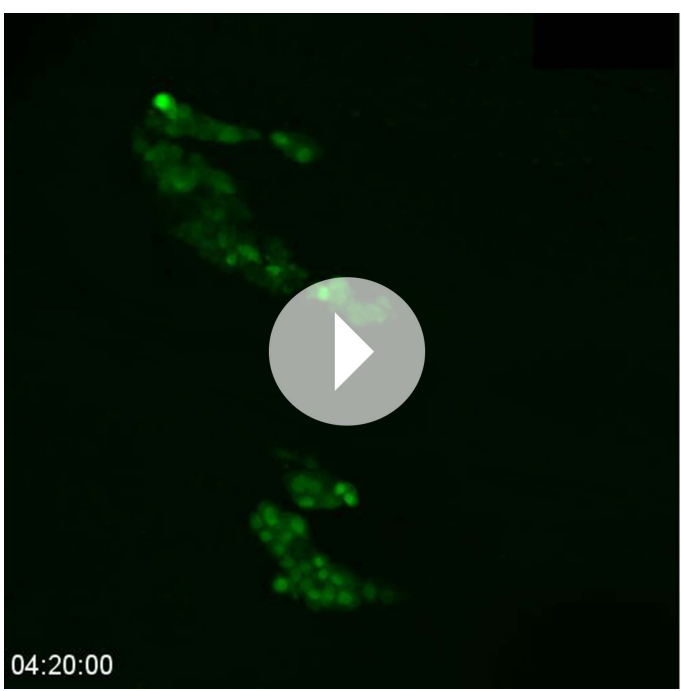

Video 8. Esg-Gal4 UAS-Stg division phase. Early divisions in a pupa overexpressing String under esgGAL4 control (genotype: esg-GAL4, UAS-nuclear GFP/UAS-stg). ADHN and PDHN indicate anterior and posterior dorsal histoblast nests. Animals were collected for imaging at $0 \mathrm{hr}$ APF. Scale bar: $50 \mu \mathrm{M}$. Refers to Figure 5-figure supplement 2.

DOI: 10.7554/eLife.07389.027

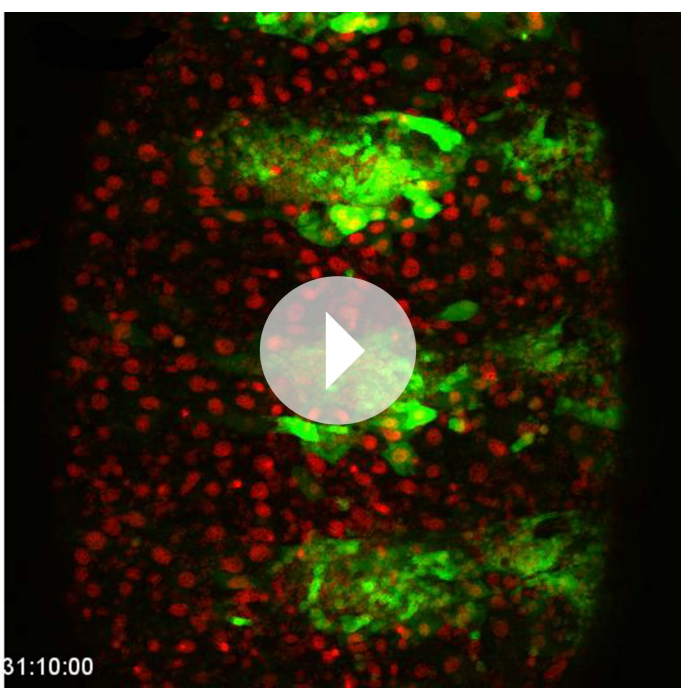

Video 9. Esg-Gal4 UAS-Stg growth phase. Growth and migration phase in a pupa overexpressing String under esg-GAL4 control, as in Video 8. ADHN and PDHN indicate anterior and posterior dorsal histoblast nests. Scale bar: $100 \mu \mathrm{M}$. Refers to Figure 5-figure supplements 2,3

DOI: 10.7554/eLife.07389.028

LF- 5' TTAGAGCTATTGCAACGAAAAGTG 3', LR- 5' GTGTAACGGGGATAATAGGATCTG 3'; the 4435 bp right arm was amplified with: RF- 5' AACACACACAGATGCAGATACAGA 3' and RR- 5' AAATAA ACGGTTCACTTCTTCTGC 3'. Following recombination, 153 bp spanning the miRNA-965 hairpin was deleted and replaced with a mini-white cassette. miR-965 mutants were crossed to heat shock-CRE flies and given heat shock treatment to excise the mini-white cassette. Deletion of miR-965 in both mutants was confirmed by genomic DNA PCR and by microRNA quantitative-PCR. All genetic tests were done using flies carrying two independently generated alleles or an allele in trans to a chromosomal deletion, $D f(2 L) E D 19$, which removes the miR-965 locus.

For generation of Rescue allele, $158 \mathrm{bp}$ of genomic region containing miR-965 hairpin was amplified with primers F- 5' GCGGGCATGTCGAGGTCGACAAGTAAAATAGCGGAATCAAAATAAT 3', R- 5' GC TCTAGAACTAGTGGATCCAACACTTTTCGTTGCAATAGCTC $3^{\prime}$ and replaced mini-white gene in miR-965 RMCE $(K O 2)$ mutant allele.

\section{Sensor and overexpression transgenic generation}

miR-965 sensor: for microRNA-GFP sensors, mature miR-965 sequence with primers F- 5' CTAGAAAGGG GAAAAGCTATACGCTTAC 3' and R- 5' TCGAGTAAGCGTATAGCTITTCCCCTTT 3' was cloned into 3'UTR of EGFP driven by tubulin promoter in pCasper4.

For UAS constructs, the miR-965 hairpin was amplified using F- 5' TATAGCGGCCGCAAGTAAAA TAGCGGAATCAAAATAAT 3' and R- 5' TATATCTAGAAACACTITCGTTGCAATAGCTC 3' and cloned into pUAST-DsRed.

\section{3'UTR reporter assays}

miR-965 expression plasmids were generated by cloning the miR-965 hairpin into pCasper-tub-SV40 with primers F- 5' TCTAGACTTTCATTTTAAGTAAAATAGCGG 3' and R- 5' CCTCGAGAACACTTTT CGTTGCAATAGCTCT 3'. For luciferase reporter constructs, 3'UTR of target genes were cloned into pCasper4-tub-Fluc-SV40 firefly luciferase vector.

The following primers were used for cloning 3'UTRs into luciferase vector.

Stg 3'UTR F- 5' GATCGCCGTGTAATTCTAGAGATGATCGTGCAGTTCGTTATC 3'. 


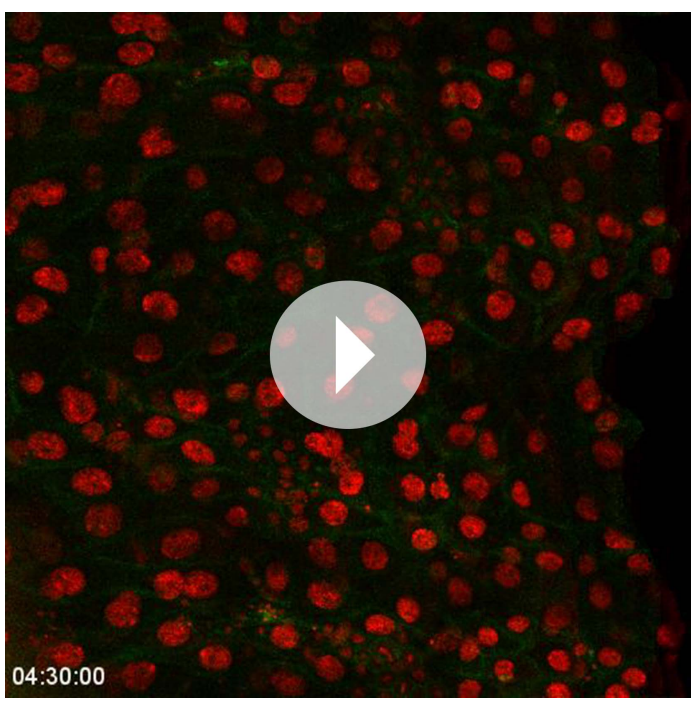

Video 10. miR-965 mutant with reduced string levels division phase. The string mutant allele, stg ${ }^{\text {EY12388 }}$ was used to reduce string levels in the miR-965 mutant (KO1/KO2) background. Atp $\alpha$-GFP (green) was used to mark cell membranes. H2-RFP (red) marks the nuclei. $\mathrm{ADHN}$ and PDHN indicate anterior and posterior dorsal histoblast nests. Scale bar: $50 \mu \mathrm{M}$. Refers to Figure 5D

DOI: 10.7554/eLife.07389.029

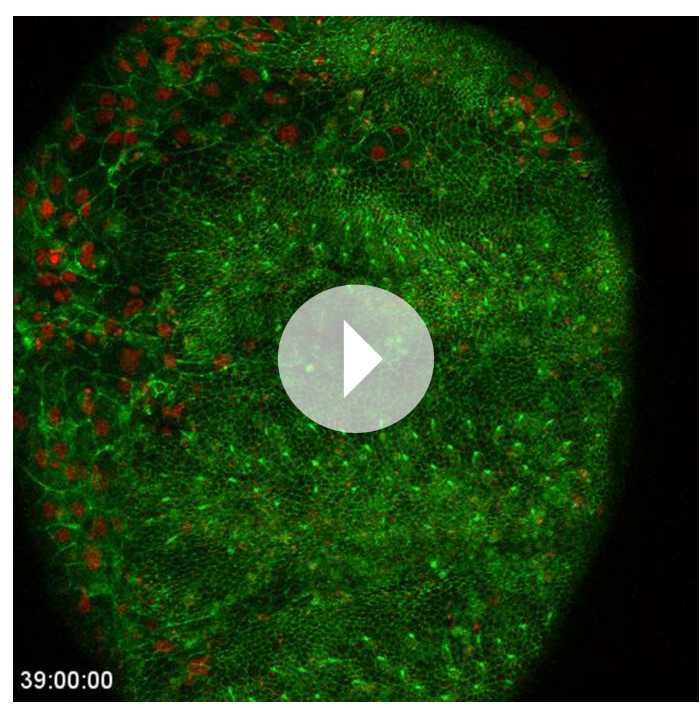

Video 11. miR-965 mutant with reduced string levels with reduced string levels growth phase. Normal growth and migration of histoblast nests in the miR-965 mutant with reduced string levels. The string mutant allele, $s t{ }^{E Y 12388}$ was used to reduce string levels in the miR-965 mutant (KO1/KO2) background. Atp $\alpha$-GFP (green) was used to mark cell membranes. H2-RFP (red) marks the nuclei. ADHN and PDHN indicate anterior and posterior dorsal histoblast nests. Scale bar: $50 \mu \mathrm{M}$. Refers to Figure 5-figure supplements 4, 5. DOI: 10.7554/eLife.07389.030

Stg 3'UTR R- 5' GGCTGCAGGTCGACCTCGAGTTCTTTTTCGTCGTGTATTAATGT 3'.

Wg 3'UTR F- 5' GATCGCCGTGTAATTCTAGACCGCCCTCTTCGTTCTTTGT 3'.

Wg 3'UTR R- 5' GGCTGCAGGTCGACCTCGAGACTCATTGTCGTTTTGTGTTTTT 3'.

Mutation in the miR-965 seed region in stg $3^{\prime}$ UTR was done using following primers:

Stg mut UTR up F- 5' GGGCGGAAAGATCGCCGTGTAATTCTAGAGATGATCGTGCAGTTCG 3'.

Stg mut UTR up R- 5' CAAATAATGATCATAAATTGTACCTAGCAGAAGTT 3'.

Stg mut UTR down F- 5' TTATGATCATTATTTGTTTATTTTTATGTAATCCG 3'.

Stg mut UTR down F- 5' ATAAACAAATAAAATTGTACCTAGCAGAAGTT 3'.

Stg extensive mut 1F- 5' GATCGCCGTGTAATTCTAGAGATGATCGTGCAGTTCGTTATC 3'.

Stg extensive mut 1R- 5' CATCACTTAGGCGTAATGTCGGATAAATAAAGTTTTATGG 3'.

Stg extensive mut 2F- 5'ACGCCTAAGTGATGCCAGATGTACCCTACTGCTAGGTACAATTTA 3'.

Stg extensive mut 2R- 5' GGCTGCAGGTCGACCTCGAGTTCTTTTTCGTCGTGTATTAATGT $3^{\prime}$.

Mutation in miR-965 seed region in wg $3^{\prime} U T R$ was done using primers:

Wg mut up F- 5' GAACTGCCTGCGTGAGATTCTCGCATGCCAGAGATCCTA 3'.

Wg mut up R- 5' CTAATAACAAAGGCTGAGTGGAGACAAAATACATAACACA 3'.

Wg mut down F- 5' TGTGTTATGTATTTTGTCTCCACTCAGCCTTTGTTATTAG 3'.

Wg mut down R- 5'GGCTGCAGGTCGACCTCGAGACTCATTGTCGTTTTGTGTTTTT 3'.

Drosophila Schneider cells (S2) were grown at $25^{\circ} \mathrm{C}$ in the absence of $\mathrm{CO} 2$, with serum free medium (SFM, Gibco) supplemented with L-Glutamine. $2 \times 10^{6} \mathrm{~S} 2$ cells were transfected in 24-well plates with $25 \mathrm{ng}$ of the firefly luciferase reporter and Renilla luciferase control plasmids, and $250 \mathrm{ng}$ of the miRNA-965 expression plasmid or empty vector. Transfection was done using Cellfectin II (Invitrogen). Transfections were performed in triplicate and each experiment was performed in at least three independent replicates. Cells were lysed 2.5 days after transfection in $100 \mu \mathrm{l}$ passive lysis buffer, shaken at room temperature for $20 \mathrm{~min}$ and dual-luciferase assays were performed according to the manufacturers protocol (Promega). 


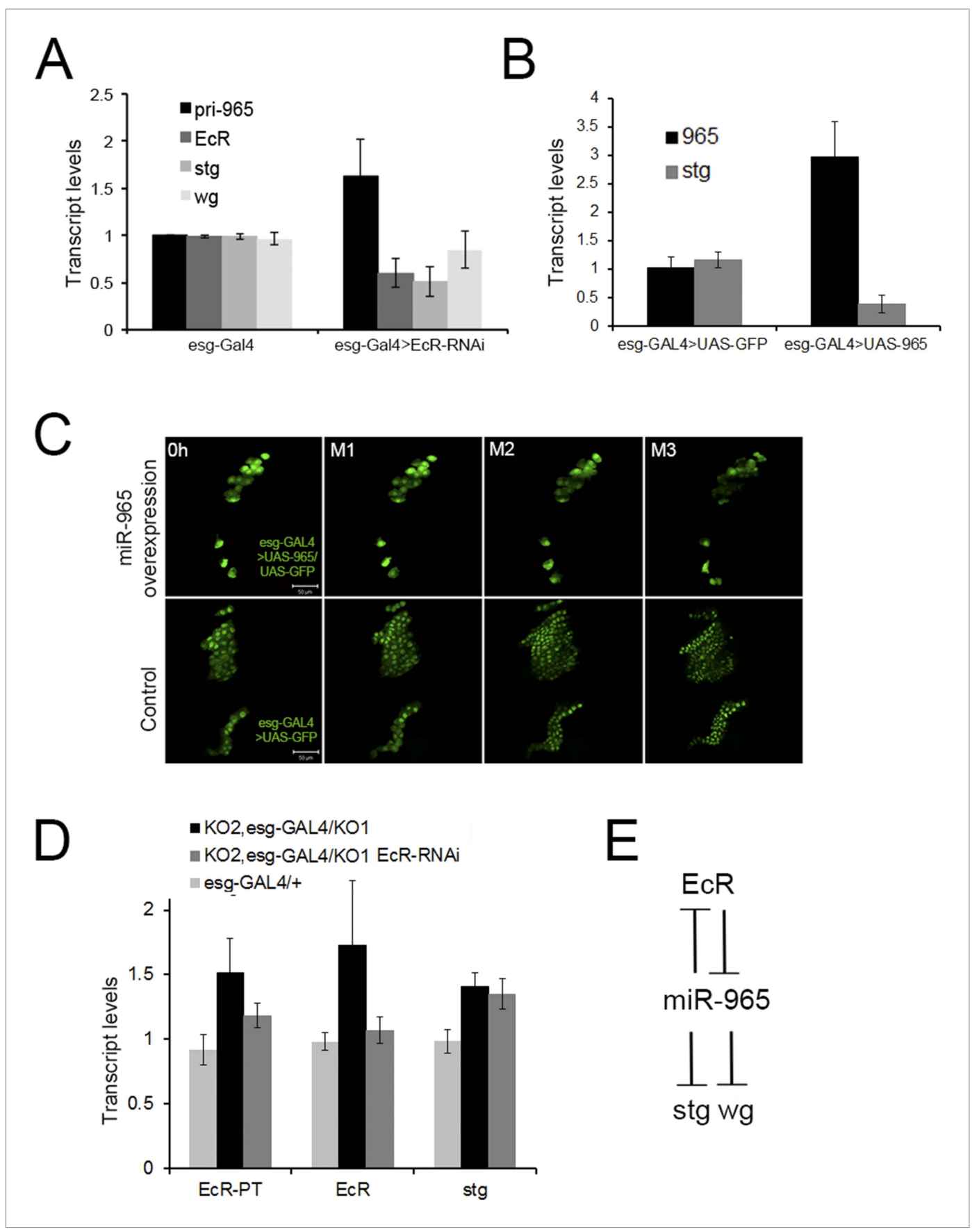

Figure 6. Regulation of miR-965 by ecdysone at the beginning of pupariation. (A) Quantitative RT-PCR showing levels of miR-965 primary transcript, ECR, and string mRNAs in RNA isolated from pupae expressing esg-GAL4 (control) and esg-GAL4 driving UAS-EcR-RNAi to deplete EcR mRNA. Samples were collected at 0 hr APF. Data were normalized to rp49 and to the esg-GAL4 control. Data represents average of three independent samples \pm SD. (B) Quantitative RT-PCR showing string mRNA in 0 hr pupae overexpressing miR-965 in histoblast cells. For quantitative microRNA PCR, data were normalized to U14, U27, SnoR422. Data were normalized to rp49 for string mRNA qPCR. Data represent the average of four independent samples \pm SD. (C) Images from time-lapse videos showing the effects of miR-965 overexpression in histoblast cells during the synchronous division phase. M1, M2 and M3 indicate three consecutive mitotic divisions in dorsal histoblast nests. Scale bar: $50 \mu \mathrm{m}$. (D) Quantitative RT-PCR showing levels of string, EcR primary transcript (EcR-PT) and mature mRNA in RNA isolated from pupae expressing esg-GAL4 (control), esg-GAL4 in the miR-965 mutant with and without UAS-EcR-RNAi to deplete EcR mRNA. esg-GAL4 was recombined onto the KO2 mutant chromosome. Samples were collected at $0 \mathrm{hr}$ APF. Data were normalized to rp49 and to the esg-GAL4 control. Data represents average of six independent samples \pm SD $p=0.37$ for stg levels between KO2, esg-GAL4/KO1 and KO2, esg-GAL4/KO1>EcR-RNAi. $p \leq 0.01$ comparing Figure 6. continued on next page 
Figure 6. Continued

primary and mature EcR transcripts between esg-GAL4 control and KO2, esg-GAL4/KO1 mutant samples. (E) Diagram of the regulatory relationships between $E_{c} R$, miR-965 and the miR-965 targets string and wg. The symbols represent repression of gene expression. miR-965 and EcR repress each other at the primary transcript level. The effect of miR-965 on EcR primary transcript is most likely indirect.

DOI: 10.7554/eLife.07389.031

The following figure supplements are available for figure 6 :

Figure supplement 1. Mature miR-965 miRNA regulation by EcR.

DOI: 10.7554/eLife.07389.032

Figure supplement 2. EcR 3' UTR reporter expression in the miR-965 mutant.

DOI: 10.7554/eLife.07389.033

\section{Quantitative RT-PCR}

Total RNA was extracted from $0 \mathrm{hr}$ or $21 \mathrm{hr}$ pupae and used for cDNA synthesis. Mature miR-965 transcript level was measured by using TaqMan miRNA assays and normalized to U14, U27 or snoR422 control primers. For target mRNA qRT-PCR, total RNA was treated with RNAse-free DNAse. First strand cDNA was synthesized using oligo-dT primers and SuperScript RT-III (Invitrogen). qRTPCR was performed using SYBR green (Applied Biosystems). Measurements were normalized to Ribosomal Protein 49. Primers:

rp49 F- GCTAAGCTGTCGCACAAA and rp49 R- TCCGGTGGGCAGCATGTG.

kis F- TTCACGGAAATCATCAAGGA and kis R- CTGTTGCTGTAGCGGATGTG stg F- ATTCTCCCATTTTCCCAGTTTT and stg R- CTTCCCATCCTATCCTTTCCTT. wg F- GTCAGGGACGCAAGCATAAT and wg R- GCGAAGGCTCCAGATAGACA. EcR F- TAACGGCCAACTGATTGTACG and EcR R- GCGGCCAAGACTTTGTTAAGA. Pri-965 F- AAATCACAAAGCAGAAGAAGTGAA and Pri-965 R- ACAGAAGGGCACATATAACGTACA.

\section{Live imaging of pupae}

For video of the division phase (0-8 hr), $0 \mathrm{hr}$ white pupa were washed with PBS, dried and stuck to imaging dishes (MatTek) with a drop of mineral oil. For growth phase videos, white pupae and allowed to age until $15-20 \mathrm{hr}$ at $25^{\circ} \mathrm{C}$. The outer cuticle was removed without damaging internal

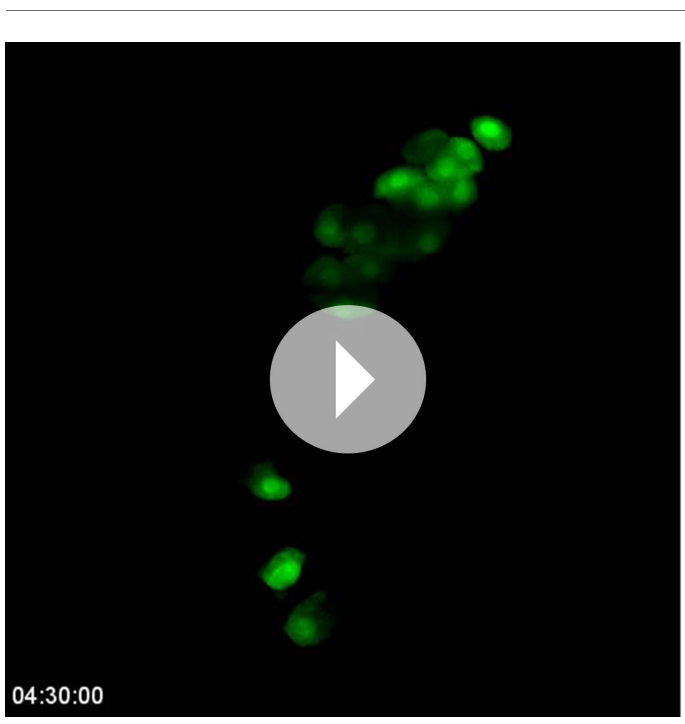

Video 12. miR-965 overexpression. Early division arrest in a pupa overexpressing miR-965 under esgGAL4 control (genotype: esg-GAL4, UAS-nuclear GFP/ UAS-miR-965). Animals were collected for imaging at $0 \mathrm{hr}$ APF. Scale bar: $50 \mu \mathrm{M}$. Refers to Figure $6 \mathrm{C}$. DOI: 10.7554/eLife.07389.034 tissues. Pupae were mounted into imaging dishes with a drop of mineral oil. Zeiss LSM700 and Leica SP5 microscopes were used for imaging and videos were processed by imageJ and photoshop. videos were taken at 5 frames/s for division phase and 10 frames/s for growth phase. For measurement of speed of migration of histoblast nests, the distance moved by the leading edges of the histoblast nests from segment 3 and 4 was measured and migration speed was calculated in micrometer/hour.

\section{Adult cuticle preparation}

Adult flies were soaked in Ethanol:Glycerol (3:1), then boiled with $10 \% \mathrm{KOH}$ at $90^{\circ} \mathrm{C}$ for $2-5 \mathrm{~min}$. Cuticles were rinsed once with PBT (PBS with $0.1 \%$ TritonX-100), then 3 times with PBS, 15 min each and mounted with glycerol.

\section{Immunohistochemistry}

For immunostaining, $0 \mathrm{hr}$ white pupae were transferred to fresh vials and raised at $25^{\circ} \mathrm{C}$ for staging. Pupae $\sim 24 \mathrm{hr}$ APF were attached to double sided tape and bisected with a blade. Gut 
and fat body were removed without disturbing the inner epithelial layer. Cuticle with attached epithelium was washed with PBS and fixed with $4 \%$ paraformaldehyde for one and half hour at $4^{\circ} \mathrm{C}$. Samples were rinsed $4 \times 15 \mathrm{~min}$ with PBT (PBS with $0.1 \%$ Triton X-100) and blocked for $2 \mathrm{hr}$ with $1 \%$ BSA in PBT. Samples were incubated with mouse anti-Wg antibody $(1: 20, D S H B)$ overnight at $4^{\circ} \mathrm{C}$. Samples were washed with PBT $(4 \times 15 \mathrm{~min})$ and incubated with AlexaFluor secondary antibody (1: 500 , Invitrogen) for $2 \mathrm{hr}$ at room temperature. Samples were washed again $4 \times 15$ min with PBT and stained with DAPI (1:1000) for $10 \mathrm{~min}$ at room temperature before mounting.

\section{Acknowledgements}

We thank David Foronda and Adam Cliff for advice on methods.

\section{Additional information}

Funding

\begin{tabular}{lll} 
Funder & Grant reference & Author \\
\hline $\begin{array}{l}\text { Agency for Science, } \\
\text { Technology and Research } \\
\text { (A*STAR) }\end{array}$ & $\begin{array}{l}\text { Institute of Molecular and } \\
\text { Cell Biology }\end{array}$ & $\begin{array}{l}\text { Pushpa Verma, Stephen M } \\
\text { Cohen }\end{array}$ \\
\hline Novo Nordisk Foundation & NNF12OC0000552 & Stephen M Cohen \\
\hline
\end{tabular}

The funders had no role in study design, data collection and interpretation, or the decision to submit the work for publication.

Author contributions

PV, Conception and design, Acquisition of data, Analysis and interpretation of data, Drafting or revising the article; SMC, Conception and design, Analysis and interpretation of data, Drafting or revising the article

Author ORCIDs

Stephen M Cohen, (iD http://orcid.org/0000-0003-2858-9163

\section{References}

Bender M, Imam FB, Talbot WS, Ganetzky B, Hogness DS. 1997. Drosophila ecdysone receptor mutations reveal functional differences among receptor isoforms. Cell 91:777-788. doi: 10.1016/S0092-8674(00)80466-3.

Bischoff M, Cseresnyes Z. 2009. Cell rearrangements, cell divisions and cell death in a migrating epithelial sheet in the abdomen of Drosophila. Development 136:2403-2411. doi: 10.1242/dev.035410.

Brennecke J, Hipfner DR, Stark A, Russell RB, Cohen SM. 2003. bantam encodes a developmentally regulated microRNA that controls cell proliferation and regulates the pro-apoptotic gene hid in Drosophila. Cell 113: 25-36. doi: 10.1016/S0092-8674(03)00231-9.

Chen YW, Weng R, Cohen SM. 2011. Protocols for use of homologous recombination gene targeting to produce MicroRNA mutants in Drosophila. Methods in Molecular Biology 732:99-120. doi: 10.1007/978-1-61779-083-6_8.

Chen YW, Song S, Weng R, Verma P, Kugler JM, Buescher M, Rouam S, Cohen SM. 2014. Systematic study of Drosophila MicroRNA functions using a collection of targeted knockout mutations. Developmental Cell 31: 784-800. doi: 10.1016/j.devcel.2014.11.029.

Edgar BA, O'Farrell PH. 1990. The three postblastoderm cell cycles of Drosophila embryogenesis are regulated in G2 by string. Cell 62:469-480. doi: 10.1016/0092-8674(90)90012-4.

Friedl P, Gilmour D. 2009. Collective cell migration in morphogenesis, regeneration and cancer. Nature Reviews. Molecular Cell Biology 10:445-457. doi: 10.1038/nrm2720.

Gauhar Z, Sun LV, Hua S, Mason CE, Fuchs F, Li TR, Boutros M, White KP. 2009. Genomic mapping of binding regions for the Ecdysone receptor protein complex. Genome Research 19:1006-1013. doi: 10.1101/gr. 081349.108.

Gautier J, Solomon MJ, Booher RN, Bazan JF, Kirschner MW. 1991. cdc25 is a specific tyrosine phosphatase that directly activates p34cdc2. Cell 67:197-211. doi: 10.1016/0092-8674(91)90583-K.

Gong WJ, Golic KG. 2003. Ends-out, or replacement, gene targeting in Drosophila. Proceedings of the National Academy of Sciences of USA 100:2556-2561. doi: 10.1073/pnas.0535280100.

Guerra M, Postlethwaite JH, Schneiderman HA. 1973. The development of the imaginal abdomen of Drosophila melanogaster. Developmental Biology 32:361-372.

Hayashi S, Hirose S, Metcalfe T, Shirras AD. 1993. Control of imaginal cell development by the escargot gene of Drosophila. Development 118:105-115. 
Herranz H, Cohen SM. 2010. MicroRNAs and gene regulatory networks: managing the impact of noise in biological systems. Genes \& Development 24:1339-1344. doi: 10.1101/gad.1937010.

Kopp A, Blackman RK, Duncan I. 1999. Wingless, decapentaplegic and EGF receptor signaling pathways interact to specify dorso-ventral pattern in the adult abdomen of Drosophila. Development 126:3495-3507.

Lawrence PA, Casal J, Struhl G. 2002. Towards a model of the organisation of planar polarity and pattern in the Drosophila abdomen. Development 129:2749-2760.

Madhavan MM, Madhavan K. 1980. Morphogenesis of the epidermis of adult abdomen of Drosophila. Journal of Embryology and Experimental Morphology 60:1-31.

Madhavan MM, Schneiderman HA. 1977. Histological analysis of the dynamics of growth of imaginal discs and histoblast nests during the larval development of Drosophila melanogaster. Roux's Archives of Developmental Biology 183:269-305.

Nakajima Y, Kuranaga E, Sugimura K, Miyawaki A, Miura M. 2011. Nonautonomous apoptosis is triggered by local cell cycle progression during epithelial replacement in Drosophila. Molecular and Cellular Biology 31:2499-2512. doi: 10.1128/MCB.01046-10.

Ninov N, Manjon C, Martin-Blanco E. 2009. Dynamic control of cell cycle and growth coupling by ecdysone, EGFR, and PI3K signaling in Drosophila histoblasts. PLOS Biology 7:e1000079. doi: 10.1371/journal.pbio.1000079.

Ninov N, Menezes-Cabral S, Prat-Rojo C, Manjon C, Weiss A, Pyrowolakis G, Affolter M, Martin-Blanco E. 2010. Dpp signaling directs cell motility and invasiveness during epithelial morphogenesis. Current Biology 20: 513-520. doi: 10.1016/j.cub.2010.01.063.

Rorth P. 2009. Collective cell migration. Annual Review of Cell and Developmental Biology 25:407-429. doi: 10. 1146/annurev.cellbio.042308.113231.

Sanicola M, Sekelsky J, Elson S, Gelbart WM. 1995. Drawing a stripe in Drosophila imaginal disks: negative regulation of decapentaplegic and patched expression by engrailed. Genetics 139:745-756.

Shirras AD, Couso JP. 1996. Cell fates in the adult abdomen of Drosophila are determined by wingless during pupal development. Developmental Biology 175:24-36. doi: 10.1006/dbio.1996.0092.

Simcox AA, Hersperger E, Shearn A, Whittle JRS, Cohen SM. 1991. Establishment of imaginal discs and histoblast nests in Drosophila. Mechanisms of Development 34:11-20. doi: 10.1016/0925-4773(91)90087-M.

Struhl G, Barbash DA, Lawrence PA. 1997. Hedgehog organises the pattern and polarity of epidermal cells in the Drosophila abdomen. Development 124:2143-2154.

Varghese J, Cohen SM. 2007. microRNA miR-14 acts to modulate a positive autoregulatory loop controlling steroid hormone signaling in Drosophila. Genes \& Development 21:2277-2282. doi: 10.1101/gad.439807.

Weng R, Chen YW, Bushati N, Cliffe A, Cohen SM. 2009. Recombinase-mediated cassette exchange provides a versatile platform for gene targeting: knockout of miR-31b. Genetics 183:399-402. doi: 10.1534/genetics.109. 105213.

Wilder EL, Perrimon N. 1995. Dual functions of wingless in the Drosophila leg imaginal disc. Development 121: 477-488. 